\title{
Compact Optical Atomic Clock Based on a Two-Photon Transition in Rubidium*
}

\author{
Kyle W. Martin, ${ }^{1}$ Gretchen Phelps, ${ }^{2}$ Nathan D. Lemke, ${ }^{2}$ Matthew S. Bigelow, ${ }^{1}$ Benjamin Stuhl, ${ }^{3}$ \\ Michael Wojcik, ${ }^{3}$ Michael Holt, ${ }^{3}$ Ian Coddington, ${ }^{4}$ Michael W. Bishop, ${ }^{2}$ and John H. Burke ${ }^{2}$ \\ ${ }^{1}$ Applied Technology Associates dba ATA, 1300 Britt Street SE Albuquerque, NM 87123 \\ ${ }^{2}$ Air Force Research Laboratory, Space Vehicles Directorate Kirtland Air Force Base, NM 87117 \\ ${ }^{3}$ Space Dynamics Laboratory, 1695 North Research Park Way North Logan, UT 84341 \\ ${ }^{4}$ National Institute of Standards and Technology, 325 Broadway Boulder, CO 80305
}

(Dated: March 28, 2019)

\begin{abstract}
Extra-laboratory atomic clocks are necessary for a wide array of applications (e.g. satellitebased navigation and communication). Building upon existing vapor cell and laser technologies, we describe an optical atomic clock, designed around a simple and manufacturable architecture, that utilizes the $778 \mathrm{~nm}$ two-photon transition in rubidium and yields fractional frequency instabilities of $3 \times 10^{-13} / \sqrt{\tau(s)}$ for $\tau$ from $1 \mathrm{~s}$ to $10000 \mathrm{~s}$. We present a complete stability budget for this system and explore the required conditions under which a fractional frequency instability of $1 \times 10^{-15}$ can be maintained on long timescales. We provide precise characterization of the leading sensitivities to external processes including magnetic fields and fluctuations of the vapor cell temperature and $778 \mathrm{~nm}$ laser power. The system is constructed primarily from commercially-available components, an attractive feature from the standpoint of commercialization and deployment of optical frequency standards.
\end{abstract}

\section{INTRODUCTION}

High stability clocks and oscillators play an integral role in many modern technologies such as navigation and communications [1]. Laboratory-based primary frequency standards, which utilize microwave transitions between atomic hyperfine levels, provide the highest degree of timing accuracy and are used to form international timescales $[2,3]$; in many cases, however, applications beyond timekeeping require clocks that are deployed outside the laboratory setting. One well-known case is that of global navigation satellite systems (GNSS), which employ space-qualified frequency standards aboard satellites in medium earth orbit and/or geosynchronous orbit $[1,4]$. While portable clocks are typically outpaced by their laboratory counterparts in terms of stability and accuracy, they nonetheless offer very low levels of frequency instabilities; in the case of rubidium atomic frequency standards, clocks are commercially available with a drift rate below $10^{-13}$ /day and a frequency noise floor less than $10^{-14}[5]$.

With the advent of fully stabilized optical frequency combs in 2000 [6-8], optical frequency standards have rapidly surpassed the capabilities of microwave clocks in both stability [9-12] and systematic uncertainty [13$15]$. Efforts to reduce the size and increase portability of these systems is an ongoing area of interest [16]. However, these improvements have yet to make an impact on more stringent definitions of portable and deployable clocks. Much of the difficulty in developing compact and environmentally robust optical frequency standards lies with the complicated laser sources and optical systems

\footnotetext{
* A contribution of AFRL, an agency of the US government, and not subject to copyright in the United States.
}

required for laser cooling and interrogating an atomic sample. Moreover, given the high quality factor (i.e. narrow spectral linewidth) of typical optical clock transitions, laser pre-stabilization to a high-finesse Fabry-Pérot cavity is generally required, which adds significant complexity to the system. Finally, optical frequency combs have historically not been sufficiently compact or robust to warrant an effort toward deployment.

Fully realized frequency standards that are compact and portable, with some applications constraining total volume to less then 30 liters, while maintaining low fractional frequency instabilities, potential for less than $1 \times 10^{-13} / \sqrt{\tau}$, are important for a plethora of non-laboratory environments. While microwave fountain clocks that incorporate lasers for cooling transitions $[17,18]$ are an ongoing research effort yielding instabilities as low as $1.4 \times 10^{-14} / \sqrt{\tau}$ they require the use of a cryogenic sapphire oscillator (CSO), restricting their use in non-traditional environments. Clock compactness with low frequency instability can be maintained only through the use of an optical oscillator. Recently, a surge of deployable microwave clocks leveraging laser cooled $\mathrm{Rb}$ have been integrated in satellite systems [19] and others utilizing vapor cell technology and laser oscillators [2022] have shown fractional frequency instability as low as $1.4 \times 10^{-13} / \sqrt{\tau}$, with potential to meet constrained size and power requirements for on orbit operation.

With these challenges in mind, we investigate an optical clock based on a two-photon transition in a hot $\mathrm{Rb}$ vapor. Two-photon transitions are attractive because they enable Doppler-free spectra [23] without the need for laser-cooling, provided two anti-parallel laser beams are used to interrogate the atomic vapor. Moreover, the twophoton transition can often be observed via a fluorescence signal that is spectrally resolvable from the probe laser; together with the large number of atoms interrogated in the vapor phase, this enables a very high signal-to- 
noise measurement of the clock transition. For the case of the Rb two-photon transition at $778 \mathrm{~nm}$, fluorescence is readily observable at $420 \mathrm{~nm}$, and stray light in the near infrared is rejected with standard optical filtering. We further benefit in the case of the $\mathrm{Rb} 5 \mathrm{~S}_{1 / 2} \rightarrow 5 \mathrm{D}_{5 / 2}$ transition from the nearby intermediate state $5 \mathrm{P}_{3 / 2}$ that is only separated by $2 \mathrm{~nm}$ from the virtual two-photon state (see Figure 1), facilitating significant atomic excitation rates at modest optical intensities [24-26]. Conveniently, $778.1 \mathrm{~nm}$ light can be produced by second harmonic generation (SHG) of $1556.2 \mathrm{~nm}$, which falls in the telecommunications C-band, allowing the use of mature laser sources and erbium fiber frequency combs [27-29]. Of particular benefit is the availability of commercial laser systems for which the fast linewidth is significantly below the natural linewidth of the excited clock state ( $\Delta \nu \approx 330 \mathrm{kHz}$ as observed at $778 \mathrm{~nm}$ ), alleviating the requirement for laser pre-stabilization to a high finesse optical cavity. There are, however, remaining challenges that persist in hot atomic vapor, two-photon clocks. Twophoton transitions have large AC Stark shifts which must be mitigated or compensated and precision temperature control is required while probing the hot atomic vapor. These two major problems need to be addressed for any suitable frequency standard based on this transition.

The appealing features motivate our investigation of an optical Rb atomic frequency standard ("O-RAFS") as a future portable clock. While our work has not yet specifically designed the system for a small footprint and low power consumption, we anticipate this should be possible. As a first step, however, it must be shown that a vapor cell-based two-photon clock can meet the demanding timing requirements needed to enable the above applications. Of particular interest is a frequency standard that can surpass existing microwave clocks by one factor of 10 in both short- and long-term stabilities, which would translate to an Allan deviation of $\sim 1 \times 10^{-13}$ at $1 \mathrm{~s}$ and $\sim 1 \times 10^{-15}$ at 1 day [30]. Previous investigations into this transition in $\mathrm{Rb}$ were carried out in the $1990 \mathrm{~s}$ [24-26] with some renewed interest recently leveraging the development of laser and frequency comb technologies [31-33]. While initial experiments showed the system to be capable of instabilities as low as $3 \times 10^{-13} / \sqrt{\tau}$ for $\tau$ from $1-2000 \mathrm{~s}$, on longer timescales the instability increased [26], and instability below $1 \times 10^{-14}$ has not been observed. Here, we extend the range of integration to longer timescales and demonstrate a corresponding reduction of long-term instability, approaching the level of $1 \times 10^{-15}$. Key to this level of performance are tight control over the vapor density [34] and laser power [26], both of which we describe in detail below, together with a full stability budget for the frequency standard.

This paper is organized as follows.

Section II describes the design of the frequency standard and measurement system. Section III provides detailed analysis of the known contributors to clock instability, with particular emphasis on the clock laserinduced AC Stark shift, collisional effects, Zeeman shifts, (a)
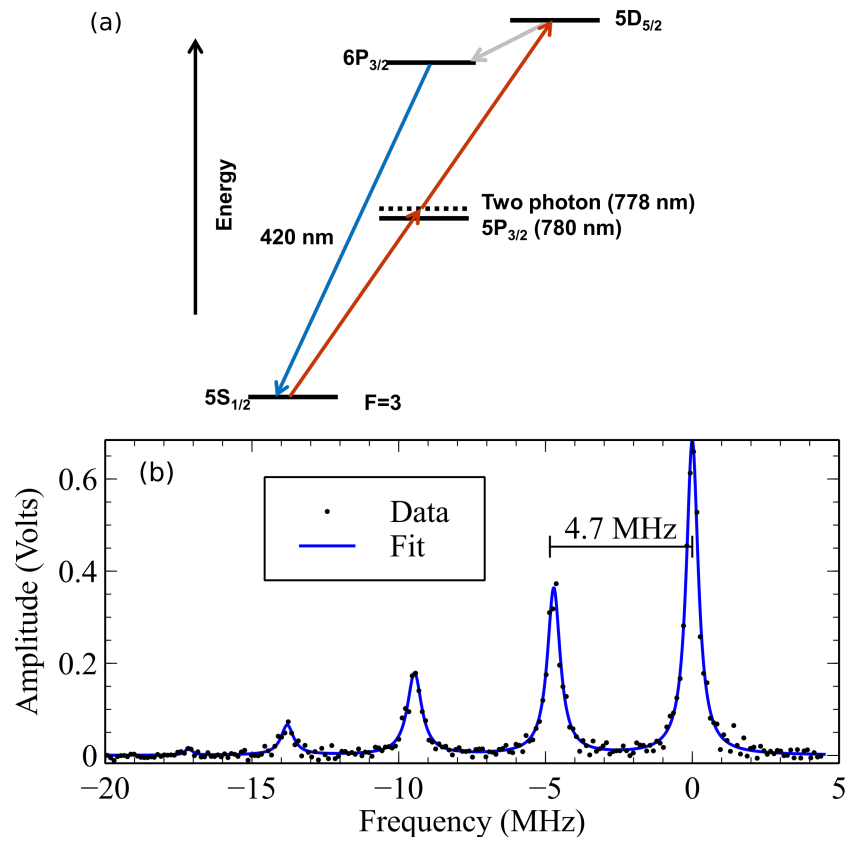

FIG. 1. (a) Partial energy level diagram of Rb. The virtual state associated with two-photon excitation at $778 \mathrm{~nm}$ is shown by the dashed line. The cascade decay path $5 \mathrm{D}_{5 / 2} \rightarrow$ $6 \mathrm{P}_{3 / 2} \rightarrow 5 \mathrm{~S}_{1 / 2}$ results in the emission of a $420 \mathrm{~nm}$ photon, which we use to observe the two-photon resonance. (b) Twophoton excitation spectrum of ${ }^{87} \mathrm{Rb}(\mathrm{F}=2)$. The frequency axis is presented as the $778 \mathrm{~nm}$ laser detuning from the $\mathrm{F}=2 \rightarrow \mathrm{F}^{\prime}=4$ transition, which has the largest Clebsch-Gordan coefficient. Included is a fit using a sum of four Lorentzian peaks of equal width and with relative peak heights and detunings constrained by the values presented in [24]. The fitting procedure results in a full-width at half-maximum linewidth of $609 \mathrm{kHz}$, which exceeds the natural linewidth by a factor of 1.8 , which we expect is due to line broadening from helium collisions [34].

and short term stability limitations. The performance of the frequency standard is detailed in Section IV, and the paper concludes with a discussion of conceived upgrades to the two-photon frequency standard in Section V.

\section{EXPERIMENTAL APPARATUS}

The design shown in Figure 2 begins with a $1556 \mathrm{~nm}$ diode laser system that produces $20 \mathrm{~mW}$ of narrow-band light, with a fast linewidth $\leq 2 \mathrm{kHz}$. This laser acts as the local oscillator for the frequency standard. A small portion of the seed laser output is sampled by a fiber splitter to form an optical beat note with a fiber frequency comb based upon the design in [29]. The fully self-referenced frequency comb coherently divides the $385 \mathrm{THz}$ optical waveform to $\sim 200 \mathrm{MHz}$, which is the comb's pulse repetition rate. After stabilization of the optical beatnote and carrier envelope offset frequency, the comb's repetition rate is photodetected and a Microsemi $5125 \mathrm{~A}$ is used as a frequency counter and the phase noise is com- 


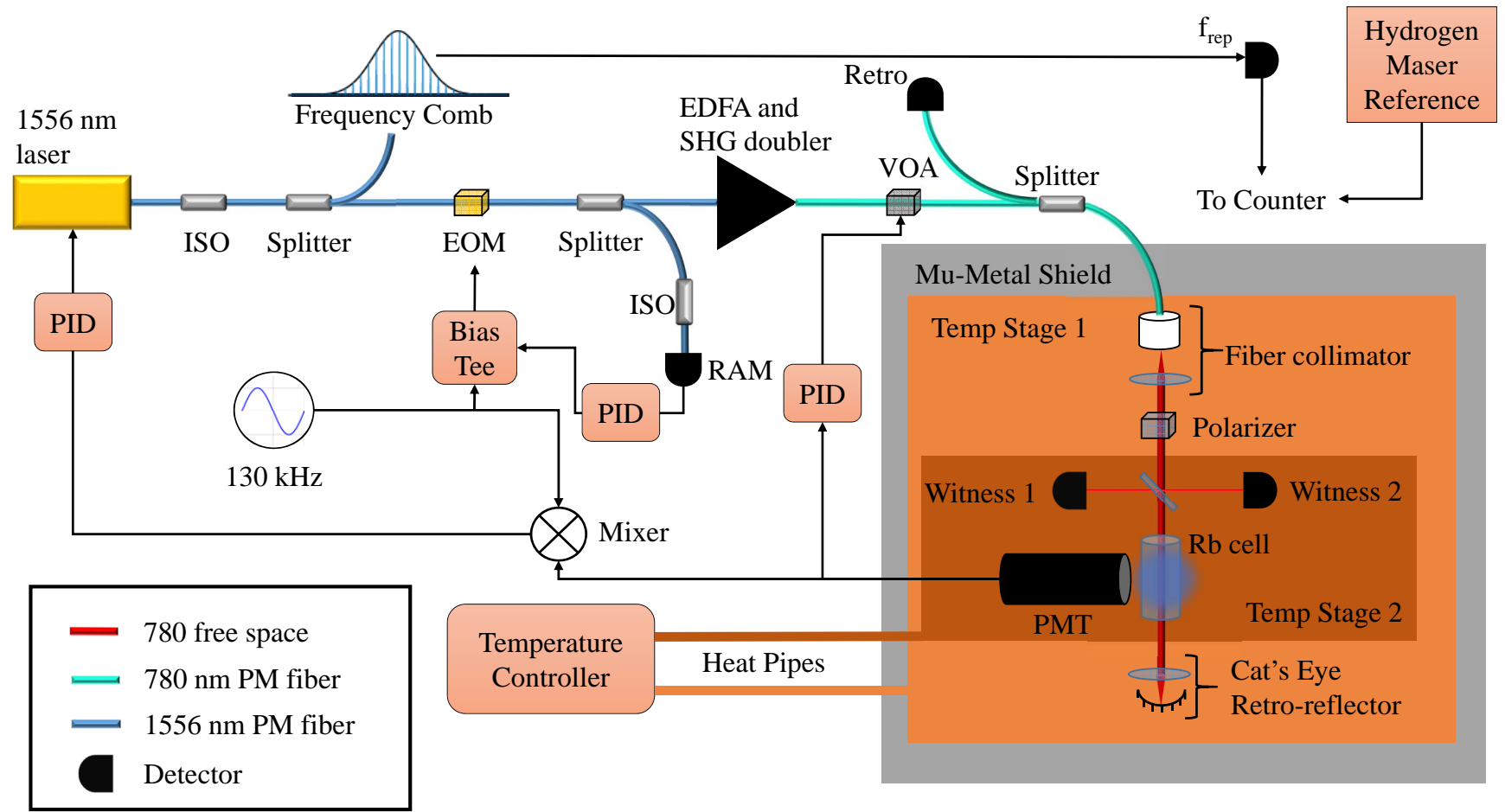

FIG. 2. An optical and simplified electrical schematic of the Rb two-photon frequency standard as described in the text. EOM electro-optic modulator; PMT - photomultiplier tube; VOA - variable optical attenuator; ISO - optical isolator; EDFA - erbium doped fiber amplifier; SHG - second harmonic generator; RAM - residual amplitude modulation; PID - proportional integral differential lock mechanism.

pared to a hydrogen maser. The remaining portion of the $1556 \mathrm{~nm}$ laser output enters a fiber-coupled electro-optic modulator (EOM) formed in a proton-exchange waveguide embedded in lithium niobate, which is driven at $130 \mathrm{kHz}$. After the EOM, the light is amplified by an erbium-doped fiber amplifier and undergoes second harmonic generation (SHG) in a PPLN crystal, outputting as much as $1 \mathrm{~W}$ of $778.1 \mathrm{~nm}$ light. The output of the SHG crystal, typically around $100 \mathrm{~mW}$, is subsequently sent through a variable optical attenuator (VOA), which is used for laser power stabilization as described below. Typically $30 \mathrm{~mW}$ of $778 \mathrm{~nm}$ light is delivered to the vapor cell assembly.

The vapor cell assembly is enclosed in a $5 \mathrm{~mm}$-thick, single layer mu-metal magnetic shield, to reduce spectral broadening associated with the Zeeman shift. The vapor cell is heated to $100{ }^{\circ} \mathrm{C}$ to generate sufficient vapor density for a high stability clock. To avoid local magnetic fields when heating the vapor cell, all heat is generated with resistive and thermo-electric devices located outside the magnetic shielding; water-filled heat pipes protrude through the magnetic shield and provide heat to the dual-zone temperature control stage surrounding the vapor cell. The vapor cell, which is a rectangular parallelepiped with dimensions $5 \times 5 \times 25 \mathrm{~mm}$, containing $>99 \%$ isotopically enriched ${ }^{87} \mathrm{Rb}$, is placed such that it has a $1 \mathrm{~K}$ thermal gradient along its length, forcing the cell's cold spot on the pinched-off fill tube of the borosilicate glass cell. The vapor cell is oriented at Brewster's angle with respect to the incident laser beam to reduce stray reflections.

The $778 \mathrm{~nm}$ laser output is delivered by polarizationmaintaining optical fiber through an opening in the magnetic shield, where it is collimated $\left(1 / \mathrm{e}^{2}\right.$ intensity radius 
$w_{0}=0.66 \mathrm{~mm}$ ) using a non-magnetic optical assembly. A calcite Glan-Taylor polarizer is placed at the output of the fiber launcher to reduce polarization wander. The laser beam is sampled by a glass plate pick-off before entering the vapor cell; un-modified Thorlabs SM05PD1A photodetectors, with dark current $20 \mathrm{nA}$ and square active area of $3.5 \mathrm{~mm}$ on a side, on each side of the glass plate monitor the optical power in the sampled beams, which remain relatively collimated with waist of $0.66 \mathrm{~mm}$. A cat's eye retro-reflector [35] provides a precisely antiparallel reflected beam, which is necessary for eliminating Doppler broadening. A portion of the fluorescence at $420 \mathrm{~nm}$ passes through a short-pass optical filter and is detected by a photomultiplier tube (PMT), dark current $5 \mathrm{nA}$. The PMT provides a fast temporal response and high electron-multiplying gain. After a transimpedance amplifier, the PMT output signal is demodulated by the $130 \mathrm{kHz}$ sinusoidal modulation applied to the EOM in a phase detector, resulting in a laser detuning-dependent error signal. A digital servo controller with dual integrators and approximately $50 \mathrm{kHz}$ bandwidth, feeds the $1556 \mathrm{~nm}$ laser's current to hold the laser on the twophoton resonance.

This design allows for the study of various parameters that contribute to the system's performance at different time scales. The short-term stability is determined by the atomic linewidth, optical intensity, detector collection efficiency, and laser frequency noise characteristics. The long-term stability, with a current goal of $<1 \times 10^{-15}$ at one day, requires the stabilization of various experimental and environmental parameters including the vapor cell temperature (Rb vapor density), magnetic field, and optical power; these parameters are investigated in subsequent sections.

\section{SOURCES OF CLOCK INSTABILITY}

In this section we discuss the leading sources of instability to the Rb two-photon system. Particular importance is paid to rigorously determining the relevant sensitivity coefficients. Because our goals call for a stable frequency standard but not necessarily one with high accuracy, we do not undertake to precisely measure the magnitude of each systematic effect, but rather to characterize the stability requirements of external parameters such as magnetic field and laser power. Table I summarizes all of the clock shifts and environmental stability parameters necessary to achieve fractional frequency instabilities of $1 \times 10^{-15}$.

\section{A. AC Stark shift}

Two-photon transitions are well-known to suffer from sizable AC Stark shifts associated with the probe laser [23]. The fractional AC Stark shift is given by
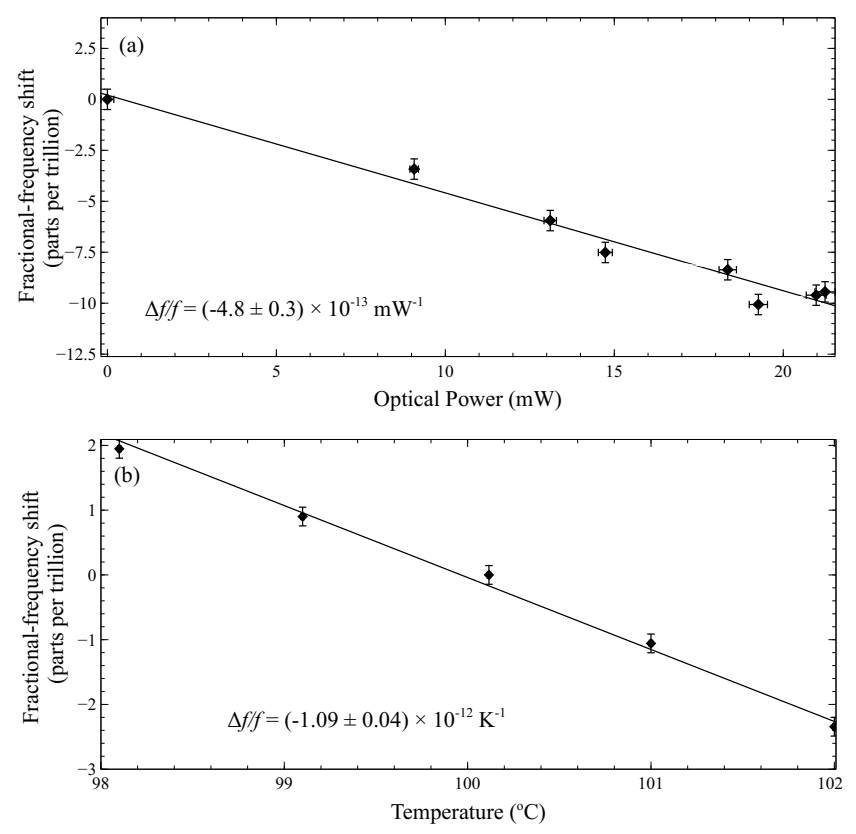

FIG. 3. (a) Experimentally measured 778 nm AC Stark shift for a $(0.66 \pm 0.05) \mathrm{mm}$ beam. The fit used was orthogonal distance regression (ODR) which weights error bars in $\mathrm{x}$ and y data yielding a reduced $\chi^{2}$ of 1.57 (b) Experimentally measured $\mathrm{Rb}$ collisional shifts, fit with ODR yielding a reduced $\chi^{2}$ of 0.908 .

$$
\frac{\Delta \nu}{\nu_{0}}=\frac{\Delta \alpha}{2 c \epsilon_{0} h} \overline{I(r)}=k\left(w_{0}\right) P
$$

where $\overline{I(r)} \propto P / w_{0}^{2}$ is the spatially-averaged laser intensity, $P$ is the one-way optical power incident on the vapor, $\nu_{0} \approx 385 \mathrm{THz}$ is the two-photon laser frequency, $w_{0}$ is the $1 / e^{2}$ intensity radius, $\Delta \alpha$ is the differential polarizability of the two clock states at $778.1 \mathrm{~nm}$ and $c, \epsilon_{0}$ and $h$ are the speed of light, permittivity of free space and Planck's constant respectively. We measured the shift experimentally utilizing the clock laser detailed in Figure 2 together with an external Ti:sapphire laser. The Ti:sapphire laser was tuned slightly away from the twophoton resonance by $2.6 \mathrm{GHz}$ to an optical frequency of $385287.8 \mathrm{GHz}$, far enough detuned to induce no measurable excitation of the vapor, yet near enough to not significantly change the polarizability. The two lasers were combined by a 50:50 beamsplitter and coupled into a single mode fiber, thereby enforcing the same spatial mode. Without changing the florescence signal size, which would contaminate the Stark shift measurement via lock point fluctuations, we varied the power of the detuned laser and measured the associated shift.

The results of this measurement are shown in Figure 3 along with a linear regression used to determine the sensitivity coefficient $k\left(w_{0}\right)$. The measured fractional clock shift coefficient is $4.8(4) \times 10^{-13} / \mathrm{mW}$ for 


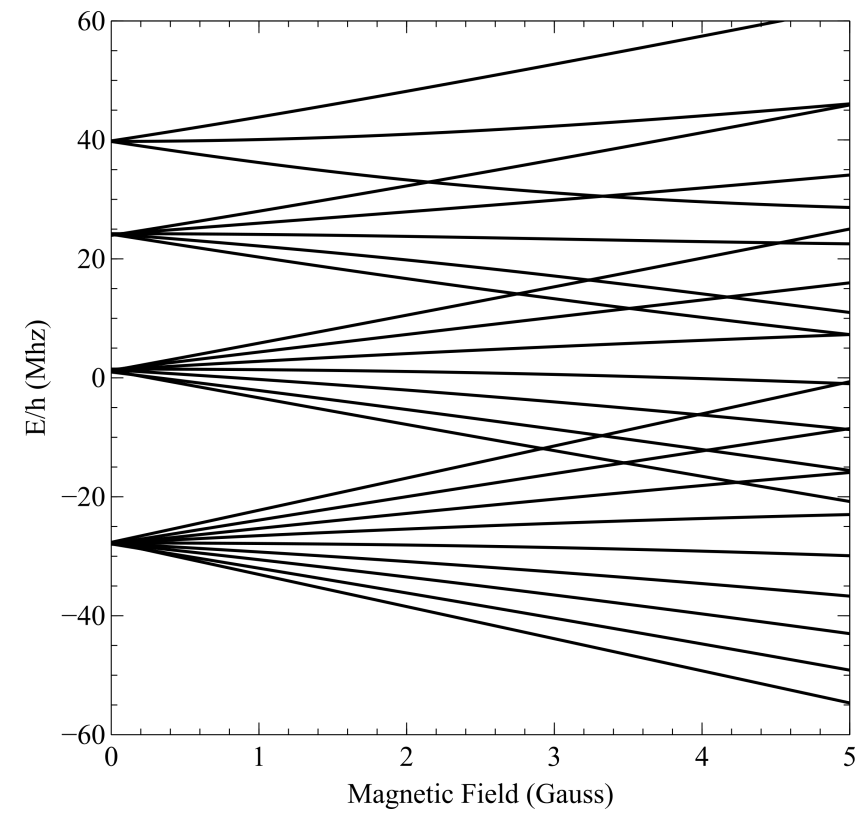

FIG. 4. Magnetic field splitting for the $5 D_{5 / 2}$ states of of ${ }^{87} \mathrm{Rb}$, which was determined by numerical diagonalization of the total Hamiltonian as described in the text.

$w_{0}=0.66(5) \mu \mathrm{m}$.

A previous measurement [26], appropriately scaled to match our beam radius, reports a coefficient of $4.5(4) \times 10^{-13} / \mathrm{mW}$, which agrees well within the error bars of the two measurements.

This coefficient indicates that the optical power must be stabilized to $2.1 \mu \mathrm{W}$ to achieve $1 \times 10^{-15}$ clock instability, requiring a precise laser power controller. A laser power stabilization circuit was constructed (Figure 2) using feedback to a fiber-optic variable optical attenuator, which supports a loop bandwidth of $1 \mathrm{kHz}$. We found it most effective to use the fluorescence signal detected on the photomultiplier tube as the laser power sensor, rather than a sampled beam measured on a photodiode, although the latter is used as an out-of-loop witness sensor. This out-of-loop data was used to determine the fractional clock limitation imposed by laser power instability as shown in Figure 5.

\section{B. Zeeman shift}

Stray magnetic fields are an important environmental variable that can produce substantial atomic frequency shifts. The magnetic field shift in the incomplete Paschen-Bach regime of the $5 S_{1 / 2}$ ground state can be analytically calculated utilizing the Briet-Rabi formula; because our spectroscopic technique does not resolve transitions between specific magnetic sublevels, we then average over all relevant $m_{I}$ and $m_{J}$ magnetic quantum numbers. This assumption results in no first-order (linear) dependence of the clock frequency on magnetic field, and is valid for local magnetic fields $\lesssim 100 \mathrm{mG}$, which is roughly the field at which Zeeman-induced linebroadening exceeds the natural linewidth of the twophoton transition. Substitution of the Landé g-factors, $g_{J}$ and $g_{I}$ and the magnetic dipole constant from Refs. $[18,36]$ yields a second order state shift of $114 \mathrm{~Hz} / \mathrm{G}^{2}$ for ${ }^{87} \mathrm{Rb}(\mathrm{F}=2)$ and $358 \mathrm{~Hz} / \mathrm{G}^{2}$ for ${ }^{85} \mathrm{Rb}(\mathrm{F}=3)$. The clock shift for the ${ }^{5} D_{5 / 2}$ excited state does not have a simple analytical solution. The Hamiltonian,

$$
\begin{aligned}
& \boldsymbol{H}=\boldsymbol{H}_{h f s}+\boldsymbol{H}_{B}^{(h f s)} \\
& \boldsymbol{H}_{h f s}=A_{h f s} \frac{\boldsymbol{I} \cdot \boldsymbol{J}}{\hbar^{2}} \\
& +B_{h f s} \frac{\frac{3}{\hbar^{2}}(\boldsymbol{I} \cdot \boldsymbol{J})^{2}+\frac{3}{2 \hbar}(\boldsymbol{I} \cdot \boldsymbol{J})-J(J+1) I(I+1)}{2 I(2 I-1) J(2 J-1)} \\
& \boldsymbol{H}_{B}^{(h f s)}=\mu_{B}\left(g_{J} \boldsymbol{J}_{\boldsymbol{z}}+g_{I} \boldsymbol{I}_{\boldsymbol{Z}}\right) B
\end{aligned}
$$

where, $\boldsymbol{I}$ is the nuclear spin, $\boldsymbol{J}$ is the total angular momentum, $\mu_{B}$ is the Bohr magneton and B is the applied magnetic field, was generated and diagonalized numerically. Substituting the magnetic dipole and quadrapole constants, $A_{h f s}$ and $B_{h f s}$, for the $5 D_{5 / 2}$ level from Ref. [24] results in a state shift of $50 \mathrm{kHz} / \mathrm{G}^{2}$ for ${ }^{87} \mathrm{Rb}(\mathrm{F}=4)$ and $190 \mathrm{kHz} / \mathrm{G}^{2}$ for ${ }^{85} \mathrm{Rb}(\mathrm{F}=5)$. Expressing the differential Zeeman sensitivities in fractional frequency units, we find the net clock shifts to be $6.5 \times 10^{-11} / \mathrm{G}^{2}$ for ${ }^{87} \mathrm{Rb}$ and $2.5 \times 10^{-10} / \mathrm{G}^{2}$ for ${ }^{85} \mathrm{Rb}$. The energy level splitting diagram for the exited state of ${ }^{87} \mathrm{Rb}$ is shown in Figure 4. With these coefficients, we can now specify the magnetic shielding requirements; for ${ }^{87} R b\left({ }^{85} R b\right)$, the magnetic field should be stable at the $3.9 \mathrm{mG}(2.0 \mathrm{mG})$ level. We installed a rectangular $\mu$-metal shield of thickness $5 \mathrm{~mm}$ for which the expected shielding factor exceeds 1000 . In practice, the shielding factor is reduced to due to openings for the heat pipes, optical fiber, electrical cabling, and photomultiplier tube, but we nonetheless expect the residual magnetic field at the vapor cell to be $\lesssim 1 \mathrm{mG}$.

\section{Collisional shift}

The vapor cell temperature was determined using a standard 100 ohm resistive temperature detector (RTD) four wire measurement, with a duplicate device for outof-loop monitoring. Two independent temperature control stages were designed, a $333 \mathrm{~K}$ plate (temperature stage 1 in Figure 2), to provide a stable reference temperature for heat transfer control, and a second, more finely controlled $373 \mathrm{~K}$ stage (temperature stage 2) upon which the vapor cell was mounted (see Figure 2). These stages were separated by four G-11 fiberglass posts to provide conductive thermal isolation. A precision temperature controller regulated a thermoelectric device and closed the temperature servo loops. Fiberglass insulation was added around the temperature control stages to reduce convective heat loss.

The collisional shift of Rydberg states of alkali metals has been a subject of research since E. Fermi formulated a 
pseudo-potential to describe the interaction of the atoms loosely bound electron with the perturber [37]. There has been a large disagreement in the literature about the measurement of the collisional shift $[26,34,38]$. We measured a collisional shift for our ${ }^{87} \mathrm{Rb}$ enriched vapor cell by varying the vapor cell temperature and measuring the clock shift of our locked laser system. For each temperature change the system was allowed to thermalize, whereupon the resulting frequency shift was measured over $300 \mathrm{~s}$.

The obtained frequency vs. temperature is plotted in Figure 3, and the fit supports a clock shift of $-1.09(4) \times$ $10^{-12} / \mathrm{K}$, which is a factor of 2 larger than reported for ${ }^{85} \mathrm{Rb}$ by Zameroski et al. [34] in a vapor cell with natural Rb. At $373 \mathrm{~K}$ the temperature must be stable to 0.92 $\mathrm{mK}$ to achieve fractional frequency stability of $1 \times 10^{-15}$. The fractional clock limitation caused by the temperature fluctuations of temperature stage 2 , measured by the outof-loop RTD, is shown in Figure 5.

\section{Other Considerations}

A frequency modulation technique utilizing a phase modulator is employed to lock the laser to the $\mathrm{Rb} 5 \mathrm{~S}_{1 / 2} \rightarrow 5 \mathrm{D}_{5 / 2}$ two photon transition. This technique is known to suffer from residual amplitude modulation (RAM) that arises when modulation sidebands are not equal in magnitude or opposite in phase [39, 40]. Zhang et al. developed a technique to suppress both inphase and quadrature RAM [41] utilizing a feedback control of the phase modulator's DC bias and temperature, respectively. We employed a similar technique using a single feedback loop to the DC bias voltage, supporting a loop bandwidth of $10 \mathrm{kHz}$, which was combined with a sinusoidal modulation signal on a bias tee as shown in Figure 2. This method yielded suppression of $>35 \mathrm{~dB}$. Additionally, we find benefit in saturating the input to the $1556 \mathrm{~nm}$ optical amplifier, which provides a passive reduction of RAM of $>5 \mathrm{~dB}$. While these two suppression mechanisms were sufficient to achieve fractional clock instabilities shown in Figure 5, further corrections to quadrature RAM could be implemented through stabilizing the temperature of the EOM to further decrease clock instabilities.

Doppler effects are largely eliminated by retroreflecting the laser beam that passes through the vapor cell. However, residual broadening related to the absorption of two-photons from the same beam remains; this contribution to the lineshape is a Gaussian function with a full-width at half-maximum of $\sqrt{8 k_{B} T \ln 2 / m c^{2}} \nu_{0} \approx$ $571 \mathrm{MHz}$ for ${ }^{87} \mathrm{Rb}$ at $T=373 \mathrm{~K}$, with $k_{B}$ the Boltzmann constant, and $m$ the atomic mass. Absorbing two photons from the same beam occurs with the same probability as absorbing one photon from each beam; however, the linewidth associated with the former process is 1000 times greater than the latter. Hence, the Doppler-broadened peak is not easily resolved, and resid- ual Doppler effects are small.

The significant tails of the Lorentzian peaks of neighboring hyperfine transitions pull the spectral lines closer together, a phenomenon known as line-pulling. The amount by which a particular transition is shifted is calculated by summing over all relevant hyperfine Lorentzians with appropriate frequencies and strengths given by Ref. [24]. The two-photon transition is shifted by $0.477 \mathrm{~Hz}$ for ${ }^{85} \mathrm{Rb}$ and $0.030 \mathrm{~Hz}$ for ${ }^{87} \mathrm{Rb}$.

Second-order Doppler broadening, taking into account first order relativistic corrections, is given by,

$$
\frac{\delta \omega}{\omega}=\frac{\bar{v}^{2}}{2 c^{2}}
$$

where $\bar{v}^{2}=8 k_{b} T / m \pi$. For $\mathrm{Rb}$ at $373 \mathrm{~K}$ the fractional clock shift is $5 \times 10^{-13}$ with a slope of $1 \times 10^{-15} / \mathrm{K}$.

The atomic vapor is immersed in a bath of electromagnetic radiation whose spectrum follows Planck's Law. In many cases, the blackbody radiation (BBR) shift can be treated as a DC Stark shift, since the radiation is far off resonance from all relevant atomic transitions [42]. However, the operational temperature of our system, $373 \mathrm{~K}$, yields a blackbody spectrum that is nearly resonant with several transitions connecting to the $5 D_{5 / 2}$ state. Farley and Wing derived this perturbation for hydrogen, helium and the alkali-metal atoms for electromagnetic radiation at $300 \mathrm{~K}$ [43]. Hilico et al. calculated, assuming a $T^{4}$ behavior, that the perturbation would yield a shift of $210 \mathrm{~Hz}$ with a local slope of $\sim 1 \mathrm{~Hz} / \mathrm{K}[26]$ at $373 \mathrm{~K}$. The fractional clock shift arising from BBR is $1.3 \times 10^{-15} / \mathrm{K}$ requiring that the blackbody source be held to temperatures more stable than $770 \mathrm{mK}$; however, atomic $\mathrm{Rb}$ transitions near resonance with the blackbody spectrum at $373 \mathrm{~K}$ possibly impact the accuracy of this estimate.

The D.C. polarizability of the $5 D_{5 / 2}$ state was measured in [44], and it exceeds that of the $5 S_{1 / 2}$ state by a factor of $\sim 50$ due to low-lying transitions to nearby levels. Using this polarizability, we calculate the fractional clock sensitivity to D.C. electric fields is $5.9 \times 10^{-15} /(\mathrm{V} / \mathrm{cm})^{2}$. The magnetic shield surrounding the vapor cell assembly also acts as a Faraday cage to prevent external electric fields from reaching the atomic vapor. However, stray charge could accumulate on the glass vapor cell itself; any resulting patch potentials need to be stable at the $0.5 \mathrm{~V}$ level.

Experimentally determined collisional shifts in [34] for various noble gases were examined to put limits on vapor cell impurities. Helium is the only gas known to permeate the vapor cell, and it produces frequency shifts of $-2.1 \mathrm{MHz} /$ Torr. Therefore, we obtain that a helium leak rate of $<3.6 \times 10^{-8}$ Torr/day must be achieved in order to achieve fractional clock instabilities below $1 \times 10^{-15}$. The cell may also be permeable to methane, which has an atmospheric composition of about three times less than helium, but the shift rate due to methane has not been measured to our knowledge. 
TABLE I. The environmental variables that impact ${ }^{87} \mathrm{Rb}$ clock performance are listed along with the corresponding fractional frequency sensitivity coefficient. The right column tabulates the stability requirement for each parameter to support a fractional frequency instability of $1 \times 10^{-15}$.

\begin{tabular}{lcr}
\hline \hline Shift & $\begin{array}{c}\text { Fractional } \\
\text { Coefficient }\end{array}$ & $\begin{array}{r}\text { Stability } \\
\text { at one day }\end{array}$ \\
\hline 778 nm AC Stark & $4.8 \times 10^{-13} / \mathrm{mW}$ & $2.1 \mu \mathrm{W}$ \\
Rb density & $1.1 \times 10^{-12} / \mathrm{K}$ & $0.92 \mathrm{mK}$ \\
Blackbody Radiation & $1.3 \times 10^{-15} / \mathrm{K}$ & $770 \mathrm{mK}$ \\
DC Stark & $5.9 \times 10^{-15} /(\mathrm{V} / \mathrm{cm})^{2}$ & $0.17(\mathrm{~V} / \mathrm{cm})^{2}$ \\
$2^{\text {nd }}$ Order Doppler & $1.0 \times 10^{-15} / \mathrm{K}$ & $1.0 \mathrm{~K}$ \\
Zeeman & $6.5 \times 10^{-11} / \mathrm{G}^{2}$ & $3.9 \mathrm{mG}$ \\
Helium Collisional & $2.7 \times 10^{-8} /$ Torr & $3.6 \times 10^{-8}$ Torr \\
\hline \hline
\end{tabular}

TABLE II. Signal parameters for the ORAFS system with $10 \mathrm{~mW}$ of input power.

\begin{tabular}{lr}
\hline \hline parameter & value \\
\hline Mixer gain $(\mathrm{g})$ & 0.41 \\
Signal to noise in $10 \mathrm{kHz}$ bandwidth & $21.7 \mathrm{kHz}$ \\
Error signal slope $(\mathrm{p})$ & $9.56 \times 10^{-8} \mathrm{~V} / \mathrm{Hz}$ \\
Voltage spectral density $\left(S_{v}\right)$ & $6.9 \times 10^{-9} \mathrm{~V}^{2} / \mathrm{Hz}$ \\
Power spectral density at $500 \mathrm{kHz}$ & $2.7 \times 10^{-27} \mathrm{~Hz} / \mathrm{Hz}^{2}$ \\
\hline \hline
\end{tabular}

\section{E. Short Term Stability}

The practical noise limit of a frequency standard is the greater of the local oscillator noise and the shot noise limit of the atoms or the photons used to detect those atoms. The Allan deviation, limited by shot noise can be written,

$$
\sigma_{y}^{(S N)}=\frac{1}{\nu_{0}} \sqrt{\frac{S_{f}}{2 \tau}}
$$

where,

$$
S_{f}=\left(\frac{g}{p}\right)^{2} \frac{S_{v}}{2}
$$

$\mathrm{g}$ is the mixer gain, $\mathrm{p}$ is the error signal slope in $\mathrm{Hz} / \mathrm{V}$, $S_{v}$ is the voltage spectral density and $\nu_{0}$ is the transition frequency [26]. For the described system the necessary parameters to calculate the shot noise limit are shown in Table II and yield a shot noise limit of $4.6 \times 10^{-13} / \sqrt{\tau}$ for a $10 \mathrm{~mW}$ of light and $2.7 \times 10^{-13} / \sqrt{\tau}$ for a $30 \mathrm{~mW}$ beam.

Although the current system is not shot noise limited the fundamental limit set by the clock laser can be written as,

$$
\sigma_{y}^{(S N)}=\frac{S_{y}^{(L O)}\left[2 f_{m}\right]}{2 \sqrt{\tau}}
$$

where, $f_{m}$ is the modulation frequency, $S_{y}^{(L O)}$ is the power spectral density of the local oscillator's fractional frequency noise [45]. The power spectral density of the seed laser used at twice the modulation frequency yields a limit of $2.6 \times 10^{-14} / \sqrt{\tau}$, shown in Figure 6 .

In principle the short term instability of the ORAFS system could be decreased by either collecting more florescence or turning up incident laser power. Figure 6 shows an instance where fluorescence collection and laser power were simultaneously increased, SNR in a $10 \mathrm{kHz}$ bandwidth increased to $210 \mathrm{kHz}$. The predicted shot noise limit for this data set was $8 \times 10^{-14} / \sqrt{\tau}$. Unfortunately, after a short time the clock begin to drift from large AC Stark shifts.

Efforts towards increasing florescence detection are ongoing, however, increasing laser power remains an unappealing option, as that would yield larger AC Stark shifts, already a daunting task to mitigate.

\section{RESULTS}

Having assessed the leading contributions to instability, we next measured the clock performance by collecting the comb repetition rate and compared the phase noise to a hydrogen maser. During data collection, the vapor cell temperature and 778 nm laser power were monitored. The phase comparison was sampled at a rate of $1 \mathrm{~Hz}$ before being converted to frequency data, from which a linear drift of $-1 \times 10^{-18} / \mathrm{s}$ was removed. Figure 5 shows the resulting total modified Allan deviation of the system, as well as the expected clock performance limitations derived from out-of-loop measurements of the cell temperature and laser power. Clock performance exceeds expected stability as calculated from laser power measurements, however, long term laser power measurements from the witness photodiode are thought to be partially influenced by room temperature fluctuations; these temperature variations lead to an overestimate of Stark shift-related clock instability. Figure 6 examines a portion of the same data, highlighted because of relative instability of the light shift was low for a period. As shown in Figure 6, the $\mathrm{Rb}$ two-photon frequency standard can operate with a fractional frequency instability of $3 \times 10^{-13} / \sqrt{\tau(s)}$ for $\tau$ from 1 s to $10,000 \mathrm{~s}$, however, complications with controlling the AC Stark shift have led to a larger research effort concentrated on timescales above $10,000 \mathrm{~s}$.

\section{CONCLUSIONS AND FUTURE OUTLOOK}

We have demonstrated our system is capable of averaging down less than $4.6 \times 10^{-15}$ at $16,000 \mathrm{~s}$. While we have only limited experimental data for timescales beyond this, we have seen that the clock instability increases on longer timescales. It appears that this performance degradation is related to the AC Stark shift, which 


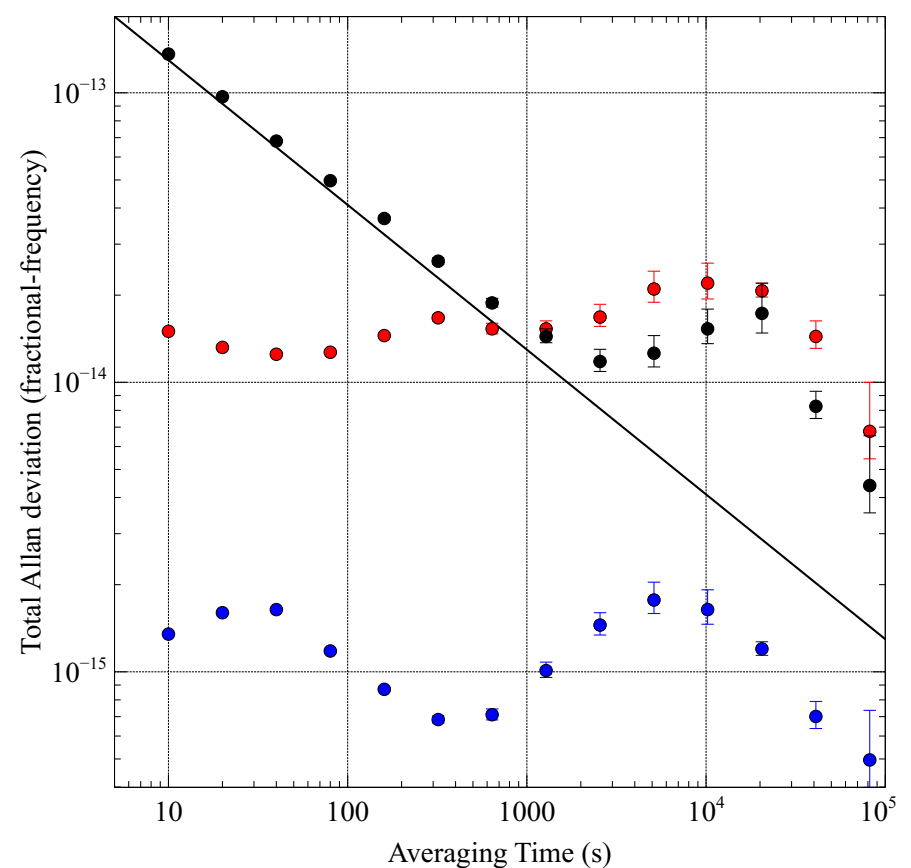

FIG. 5. The fractional frequency instability plotted as a Total Allan deviation for ${ }^{87} \mathrm{Rb}$ with $1 / \sqrt{\tau}$ white noise (black) as well as anticipated limits on the clock stability arising from cell temperature fluctuations (blue) and laser power fluctuations (red). We believe the instability limit arising from laser power fluctuations is an overestimate due to temperature-dependent effects in the witness photodiode as described in the main text

would indicate that tighter control and better measurement of the laser power is required to achieve fractional frequency instabilities of $1 \times 10^{-15}$ at one day. The initial optical design of the vapor cell assembly implemented a photodiode for use in laser power stabilization that was thermally anchored to temperature stage 2 for reduction of temperature influenced drifts. In some respects, the PMT used for fluorescence detection offers an improved measure of the average laser power across the atomic cloud because it relies on an atomic based signal rather than a beam sampling optic, for which the reflectivity is subject to polarization and temperature variations.

Nevertheless, thermal considerations are still required, as the PMT's conversion efficiency is also temperature dependent [46], thought to be believed to be somewhere between $0.5 \%$ to $1 \%$ per $\mathrm{K}$. Within the current design of the vapor cell assembly, an attempt was made to thermally connect the PMT to temperature stage 2, and fiberglass insulation was used to reduce thermal parasitics. However, temperature gradients are difficult to minimize over the $10 \mathrm{~cm}$ length of the PMT. Additionally, the PMT's high voltage power supply and transimpedance amplifier need to provide constant voltage and gain, respectively, in order to use the PMT as a longterm laser power sensor. Alternatively, if tighter control of laser power proves too challenging, there are several design modifications that allow the two-photon clock to

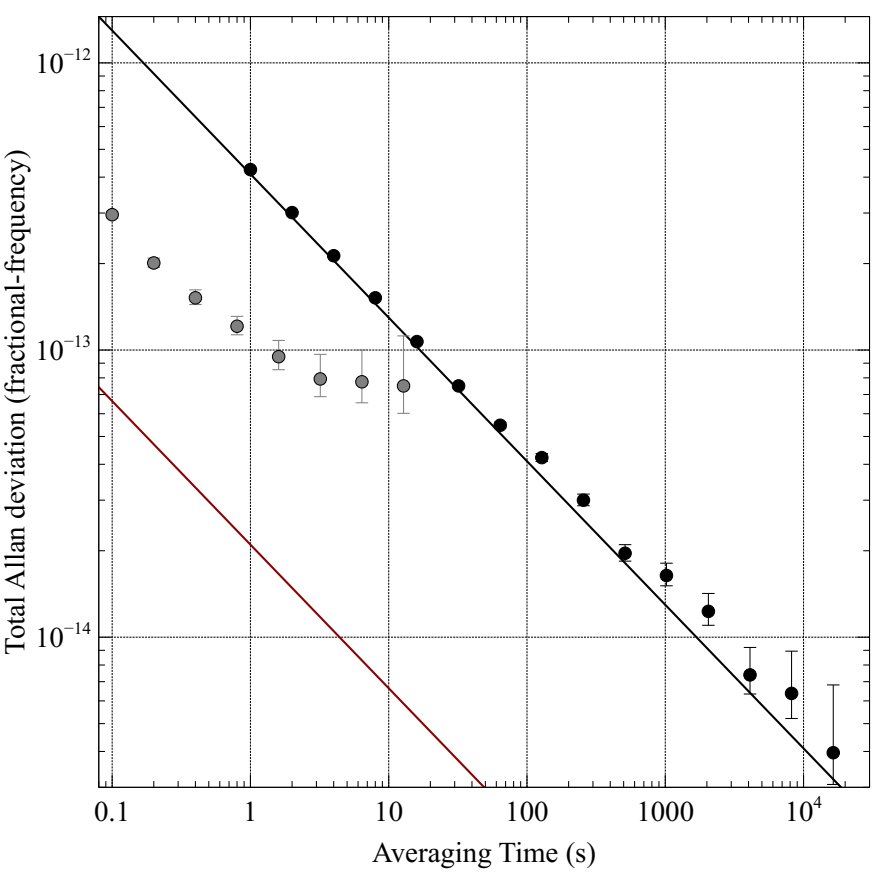

FIG. 6. The fractional frequency instability plotted as a Total Allan deviation for ${ }^{87} \mathrm{Rb}$ with $1 / \sqrt{\tau}$ white noise for a smaller section of the data presented in Figure 5 (black). Also shown: A second data set with a larger signal to noise ratio (grey) and the intermodulation limit for the laser system described in Section II (red).

operate with a smaller Stark shift. First, the spectral linewidth could be decreased by removing the helium contamination in the vapor cell; in our current cells, which are expected to be saturated with 4 mTorr of helium, the linewidth is approximately a factor of 2 larger than the natural linewidth. Removing the helium (through the use of a vacuum chamber or a helium-resistant cell [47]) would allow for a reduced laser power by a factor of 2 without affecting the short-term stability. Additional modifications include increasing the efficiency of the fluorescence detection; increasing the effective length of the vapor cell by routing the beam through the vapor on multiple non-overlapping passes; increasing the vapor density; and decreasing the desired short-term stability metric. Another final option is to increase the laser beam radius and laser power together; increasing both the power $P$ and the intensity radius $w_{0}$ by a factor of $F$ results in the same photon detection rate (accounting for both the decreased optical intensity and the increased number of atoms in the beam path) but a smaller overall AC Stark shift by the same factor $F$.

Beyond improvements to the stability, future work with this system necessarily involves miniaturizing and hardening the laser and frequency comb for future field deployment. Particular emphasis will need to be paid to automation of the locking electronics and simplification of the control loop architecture, which currently includes 
10 independent temperature control segments. Reducing the power consumption of the frequency comb, which currently uses four pump lasers, is another important area of research. Finally, any attempt to prepare this type of advanced optical clock for space will have to overcome the challenge of radiation-induced darkening of optical components. Despite these challenges, we remain optimistic that a vapor cell optical clock, such as the one described here, could soon be deployed to provide precise timekeeping for a host of applications.

\section{ACKNOWLEDGMENTS}

We thank Jordan Armstrong and the Space Dynamics Laboratory machine shop for assistance in constructing the experimental apparatus. We thank Steve Lipson for careful reading of the manuscript.
[1] L. Maleki and J. Prestage, "Applications of clocks and frequency standards: from the routine to tests of fundamental models," Metrologia 42, S145 (2005).

[2] R. Wynands and S. Weyers, "Atomic fountain clocks," Metrologia 42, S64 (2005).

[3] T. E. Parker, S. R. Jefferts, T. P. Heavner, and E. A. Donley, "Operation of the NIST-F1 caesium fountain primary frequency standard with a maser ensemble, including the impact of frequency transfer noise," Metrologia 42, 423-430 (2005).

[4] J. G. McNeff, "The global positioning system," IEEE Transactions on Microwave Theory and Techniques 50, 645-652 (2002).

[5] V. Formichella, J. Camparo, and P. Travella, "Influence of the ac-Stark shift on GPS atomic clock timekeeping," Applied Physics Letters (2017).

[6] D. J. Jones, S. A. Diddams, J. K. Ranka, A. Stentz, R. S. Windeler, J. L. Hall, and S. T. Cundiff, "Carrierenvelope phase control of femtosecond mode-locked lasers and direct optical frequency synthesis," Science 288, 635-639 (2000).

[7] S. A. Diddams, D. J. Jones, J. Ye, S. T. Cundiff, J. L. Hall, J. K. Ranka, R. S. Windeler, R. Holzwarth, T. Udem, and T. W. Hansch, "Direct link between microwave and optical frequencies with a $300 \mathrm{THz}$ femtosecond laser comb," Physical Review Letters 84, 5102-5105 (2000).

[8] R. Holzwarth, T. Udem, T. W. Hansch, J. C. Knight, W. J. Wadsworth, and P. S. J. Russell, "Optical frequency synthesizer for precision spectroscopy," Physical Review Letters 85, 2264-2267 (2000).

[9] N. Hinkley, J. A. Sherman, N. B. Phillips, M. Schioppo, N. D. Lemke, K. Beloy, M. Pizzocaro, C. W. Oates, and A. D. Ludlow, "An atomic clock with $10^{-18}$ instability," Science 341, 1215-1218 (2013).

[10] B. J. Bloom, T. L. Nicholson, J. R. Williams, S. L. Campbell, M. Bishof, X. Zhang, W. Zhang, S. L. Bromley, and J. Ye, "An optical lattice clock with accuracy and stability at the $10^{-18}$ level," Nature 506, 71-75 (2014).

[11] J. Lodewyck, S. Bilicki, E. Bookjans, J.-L. Robyr, C. Shi, G. Vallet, R. Le Targat, D. Nicolodi, Y. Le Coq, J. Guna, M. Abgrall, P. Rosenbusch, and S. Bize, "Optical to

\section{REFERENCES}

microwave clock frequency ratios with a nearly continuous strontium optical lattice clock," Metrologia 53, 1123 (2016).

[12] I. R. Hill, R. Hobson, W. Bowden, E. M. Bridge, S. Donnellan, E. A. Curtis, and P. Gill, "A low maintenance Sr optical lattice clock," in 8th symposium on frequency standards and metrology 2015, Journal of Physics: Conference Series No. 012019 (2016).

[13] T. L. Nicholson, S. L. Campbell, R. B. Hutson, G. E. Marti, B. J. Bloom, R. L. McNally, W. Zhang, M. D. Barrett, M. S. Safronova, G. F. Strouse, W. L. Tew, and J. Ye, "Systematic evaluation of an atomic clock at $2 \times$ $10^{-18}$ total uncertainty," Nat. Commun. 6, 6896- (2015).

[14] N. Huntemann, C. Sanner, B. Lipphardt, Chr. Tamm, and E. Peik, "Single-ion atomic clock with $3 \times 10^{-18}$ systematic uncertainty," Phys. Rev. Lett. 116, 063001 (2016).

[15] C. W. Chou, D. B. Hume, J. C. J. Koelemeij, D. J. Wineland, and T. Rosenband, "Frequency comparison of two high-accuracy $\mathrm{Al}^{+}$optical clocks," Physical Review Letters 104, 070802 (2010).

[16] S. B. Koller, J. Grotti, St. Vogt, A. Al-Masoudi, S. Dörscher, S. Häfner, U. Sterr, and Ch. Lisdat, "Transportable optical lattice clock with $7 \times 10^{-17}$ uncertainty," Phys. Rev. Lett. 118, 073601 (2017).

[17] J. Guena, M. Abgrall, D. Rovera, P. Laurent, B. Chupin, M. Lours, G. Santarelli, P. Rosenbusch, M. E. Tobar, R. Li, K. Gibble, A. Clairon, and S. Bize, "Progress in atomic fountains at LNE-SYRTE," IEEE Transactions on Ultrasonics, Ferroelectrics, and Frequency Control 59, 391-409 (2012).

[18] S. Bize, Y. Sortais, M. S. Santos, C. Mandache, A. Clairon, and C. Salomon, "High-accuracy measurement of the ${ }^{87} \mathrm{Rb}$ ground-state hyperfine splitting in an atomic fountain," Europhysics Letters 45 (1999).

[19] L. Liu, D. L, W. Chen, T. Li, Q. Qu, B. Wang, L. Li, W. Ren, Z. Dong, J. Zhao, W. Xia, X. Zhao, J. Ji, M. Ye, Y. Sun, Y. Yao, D. Song, Z. Liang, S. Hu, D. Yu, X. Hou, W. Shi, H. Zang, J. Xiang, X. Peng, and Y. Wang, "Test of cold atom clock in orbit," arXiv:1709.03256 (2017).

[20] P. Yun, F. Tricot, C. E. Calosso, S. Micalizio, B. François, R. Boudot, S. Guérandel, and E. de Clercq, "High- 
performance coherent population trapping clock with polarization modulation," Phys. Rev. Applied 7, 014018 (2017).

[21] A. Godone, F. Levi, C. E. Calosso, and S. Micalizio, "High-performing vapor-cell frequency standards," La Rivista del Nuovo Cimento 38, 133 (2015).

[22] S. Micalizio, C. E. Calosso, A. Godone, and F. Levi, "Metrological characterization of the pulshigh Rb clock with optical detection," Metrologia 49, 425 (2012).

[23] G. Grynberg and B. Cagnac, "Doppler-free multiphotonic spectroscopy," Reports on Progress in Physics 40, 791-841 (1977).

[24] F. Nez, F. Biraben, R. Felder, and Y. Millerioux, "Optical frequency determination of the hyperfine components of the $5 \mathrm{~S}_{1 / 2} \rightarrow 5 \mathrm{D}_{5 / 2}$ two-photon transitions in rubidium," Optics Communications 102, 432-438 (1993).

[25] D Touahri, O Acef, A Clairon, J.-J Zondy, R Felder, L Hilico, B de Beauvoir, F Biraben, and F Nez, "Frequency measurement of the $5 \mathrm{~S}_{1 / 2}(\mathrm{f}=3) \rightarrow 5 \mathrm{D}_{5 / 2}(\mathrm{f}=5)$ two-photon transition in rubidium," Optics Communications 133, 471 - 478 (1997).

[26] L. Hilico, R. Felder, D. Touahri, O. Acef, A. Clairon, and F. Biraben, "Metrological features of the rubidium twophoton standards of the BNM-LPTF and Kastler Brossel Laboratories," Eur. Phys. J. AP 4, 219-225 (1998).

[27] N. Kuse, C.-C. Lee, J. Jiang, C. Mohr, T. R. Schibli, and M.E. Fermann, "Ultra-low noise all polarizationmaintaining Er fiber-based optical frequency combs facilitated with a graphene modulator," Opt. Express 23, 24342-24350 (2015).

[28] M. Lezius, T. Wilken, C. Deutsch, M. Giunta, O. Mandel, A. Thaller, V. Schkolnik, M. Schiemangk, A. Dinkelaker, A. Kohfeldt, A. Wicht, M. Krutzik, A. Peters, O. Hellmig, H. Duncker, K. Sengstock, Windpassinger. P., K. Lampmann, T. Hülsing, T. W. Hänsch, and R. Holzwarth, "Space-borne frequency comb metrology," Optica 3, 1381-1387 (2016).

[29] L. C. Sinclair, J.-D. Deschnes, L. Sonderhouse, W. C. Swann, I. H. Khader, E. Baumann, N. R. Newbury, and I. Coddington, "Invited article: A compact optically coherent fiber frequency comb," Review of Scientific Instruments 86, 081301 (2015), http://dx.doi.org/10.1063/1.4928163.

[30] T. Schuldt, K. Döringshoff, E. V. Kovalchuk, A. Keetman, J. Pahl, A. Peters, and C. Braxmaier, "Development of a compact optical absolute frequency reference for space with $10^{-15}$ instability," Appl. Opt. 56, 11011106 (2017).

[31] Adela Marian, Matthew C. Stowe, John R. Lawall, Daniel Felinto, and Jun Ye, "United time-frequency spectroscopy for dynamics and global structure," Science 306, 2063-2068 (2004).

[32] Jiutao Wu, Dong Hou, Zhengyu Qin, Zhigang Zhang, and Jianye Zhao, "Observation of Rb two-photon absorption directly excited by an erbium-fiber-laser-based optical frequency comb via spectral control," Phys. Rev. A 89, 041402 (2014).
[33] K. D. Rathod and V. Natarajan, "Accessing the $5 \mathrm{~S}_{1 / 2} \rightarrow 5 \mathrm{D}_{5 / 2}$ two-photon transition in $\mathrm{Rb}$ using a diode laser system," ArXiv e-prints (2015), arXiv:1511.05100 [physics.optics].

[34] N. D. Zameroski, G. D. Hager, C. J. Erickson, and J. H. Burke, "Pressure broadening and frequency shift of the $5 \mathrm{~S}_{1 / 2} \rightarrow 5 \mathrm{D}_{5 / 2}$ and $5 \mathrm{~S}_{1 / 2} \rightarrow 7 \mathrm{~S}_{1 / 2}$ two photon transitions in ${ }^{85} \mathrm{Rb}$ by the noble gases and $\mathrm{n}_{2}$," Journal of Physics B: Atomic, Molecular and Optical Physics 47, 225205 (2014).

[35] J. J. Snyder, "Paraxial ray analysis of a cat's-eye retroreflector," Appl. Opt. (1975).

[36] E. Arimondo, M. Inguscio, and P. Violino, "Experimental determinations of the hyperfine structure in the alkali atoms," Reviews of Modern Physics 49 (1977).

[37] Enrico Fermi, "Sopra lo spostamento per pressione delle righe elevate delle serie spettrali," Il Nuovo Cimento (1924-1942) 11, 157 (2008).

[38] Osama Terra and Hatem Hussein, "An ultra-stable optical frequency standard for telecommunication purposes based upon the $5 \mathrm{~s} 1 / 25 \mathrm{~d} 5 / 2$ two-photon transition in rubidium," Applied Physics B (2016).

[39] G. C. Bjorklund, "Frequency-modulation spectroscopy: a new method for measuring weak absorptions and dispersions," Opt. Lett. 5, 15 (1980).

[40] Long-Sheng Ma Jun Ye and John L. Hall, "Ultrasensitive detections in atomic and molecular physics: demonstration in molecular overtone spectroscopy," Opt. Lett. 15, 6 (1998).

[41] W. Zhang, M. J. Martin, C. Benko, J. L. Hall, J. Ye, C. Hagemann, T. Legero, U. Sterr, F. Riehle, G. D. Cole, and M. Aspelmeyer, "Reduction of residual amplitude modulation to $1 \times 10^{-6}$ for frequency modulation and laser stabilization," Opt. Lett. 39, 1980-1983 (2014).

[42] Wayne M. Itano, L. L. Lewis, and D. J. Wineland, "Shift of ${ }^{2} \mathrm{~S}_{1 / 2}$ hyperfine splittings due to blackbody radiation," Phys. Rev. A 25, 1233-1235 (1982).

[43] J. W. Farley and W. H. Wing, "Accurate calculation of dynamic stark shifts and depopulation rates of rydberg energy levels induced by blackbody radiation. hydrogen, helium, and alkali-metal atoms," Phys. Rev. A (1981).

[44] D. Tregubov, S. Snigirev, A. Golovizin, S. Pyatchenkov, D. Sukachev, A. Akimov, V. Sorokin, and N. Kolachevskyv, "Measurement of the 5D level polarizabilities in laser cooled Rb atoms," Journal of Physics : Conference Series 635 (2015).

[45] C. Audoin, V. Candelier, and N. Dimarcq, "A limit to the frequency stability of passive frequency standards," in Precision Electromagnetic Measurements, 1990. CPEM '90 Digest., Conference on (1990) pp. 93-.

[46] A. T. Young, "Temperature effects in photomultipliers and astronomical photometry," Applied Optics 2, 51 (1963).

[47] A. T. Dellis, V. Shah, E. A. Donley, S. Knappe, and J. Kitching, "Low helium permeation cells for atomic microsystems technology," Opt. Lett. 41, 2775-2778 (2016). 